\title{
Marianne Akerberg, (comp.) Adquisición de segundas lenguas. Estudios y perspectivas. México, CELE-UNAM, 2005. (Colec. Líneas de Investigación en el Departamento de Lingüística Aplicada. No. 3)
}

Adquisición de segundas lenguas. Estudiosy perspectivas está conformado por siete trabajos que ponen en la mesa temas como lo son: el bilingüismo; la percepción auditiva en L2; la adquisición de aspectos morfológicos relacionados con el tiempo, el aspecto y el caso; el desarrollo de estrategias, y la adquisición del vocabulario. Temas de gran actualidad que conducen a reflexiones y discusiones por los puntos tan polémicos que abordan, relacionados con lo que implica "aprender" otro idioma en la era de la globalización.

Abre la obra el prólogo signado por la Dra. María Eugenia Herrera, quien señala que "la obra ofrece datos teóricos y empíricos valiosos para comprender fenómenos complejos de codificación y decodificación lingüística, asimismo, nos dice que "la obra ofrece diferentes perspectivas y planteamientos metodológicos de interés para el investigador, el profesor y el al umno de lingüística aplicada, así como también para el maestro de lenguas extranjeras".

Marianne Akerberg, pondera la importancia del aprendizaje de otra u otras lenguas extranjeras si se quiere tener movilidad por el mundo. Señala, además, que en "este libro se reúnen varios trabajos sobre adquisición de una segunda lengua (L2), que contribuyen a ampliar el horizonte de este tema según los planteamientos más aceptados en la actual idad".

En el artículo "Bilingüismo", cognición y metacognición, AlineSignoret presenta un estudio confirmatorio, experimental y transversal sobre la relación causal entre el bilingüismo escolar, coordinado, completo, aditivo e igualitario, y el desarrollo de la metacognición o conciencia lingüística del niño a nivel preescolar.

Además de señalar la importancia del estudio del bilingüismo y su relación con la cognición y la metacognición, muestra la preocupación de su autora por definir lo complejo del término. Para realizar esta tarea, elabora un cuadro en el que presenta una tipología de bilingüismo. Los criterios que establece son: neurológicos, lingüísticos, la edad durante laadquisición, el contextofísico-social y factores psicosociales.

Termina la autora el artículo con resultados de su investigación, subrayando que los datos obtenidos demuestran que el bilingüismo es un promotor delacognición en el niño 
preescolar y un desencadenante de la adquisición de la palabra. De esta manera, confirma la hipótesis del bilingüismo escolar como un acelerador de la cognición y más específicamente, de la reflexividad de la palabra".

Como profesores de lenguas extranjeras, y concretamente del español, como es mi caso, es sintomático escuchar comunicaciones deficientes, por ejemplo, entre un hablante de japonés y un estadounidense porque cada quien percibe la lengua española desde los conocimientos de la propia. Es precisamente este problema el que desarrolla Marianne Akerberg en el espléndido artículo "La percepción auditiva como factor en la adquisición de sonidos de L2". Ella señala que "hoy los investigadores están convencidos de que la percepción es una condición para la buena pronunciación".

Su propuesta fundamental, basada en varios autores como Goodman y Scovel, es presentar la necesidad de una teoría de percepción auditiva. Llega a esta conclusión después de real izar una investigación con estudiantes mexicanos que estudian portugués en la UNAM.

En el desarrollo de la percepción auditiva en la lengua 1 , nos dice, que para llegar a tener la capacidad de reconocer los sonidos importantes de la lengua materna, Ios fonemas, el camino es largo y arduo y que en las investigaciones sobre el desarrollo dela percepción auditiva en la L1, normalmente se habla de que la unidad percibidaes un segmento fonético. Ciertas propuestas consideran la sílaba como la unidad más adecuada, otras más indican que es importante consi derar los rasgos suprasegmentales: ritmo, acento y entonación y otros estudiosos más como Goodman, incluyen la prosodia.

En el desarrollo de la percepción auditiva en L2 por adultos, con una estructura cognitiva más desarrollada, ya disponen de todo el sistema lingüístico y no dependen solamente de lo que captan los senti dos. Por eso usan también, otros niveles lingüísticos en la percepción, como el léxico semánticoy el sintáctico, y no sólo los básicos, el auditivo y el fonético.

Así, plantea que los investigadores, sin importar la corriente lingüística a la que pertenezcan, están de acuerdo en que la adquisición del sistema fonológico de la L2, pasa por la percepción auditiva de un oído que ha sido entrenado para prestar atención a las categorías de los sonidos importantes de la primera lengua.

Después de presentar las propuestas de varios autores, Marianne Akerber Ilega a la conclusión de que todos los problemas de producción se pueden atribuir a una deficiente percepción y que, el problema no radica en la dificultad en escuchar los elementos, sino en la interiorización o automatización de las regl as de las restricciones fonotácticas.

"Tiempo y aspecto en la adquisición de la gramática" de Marilyn Buck, esun trabajo que da cuenta de uno de los eternos problemas en la enseñanza de lengua extranjeras relacionado con el tiempo verbal y el aspecto, términos referidos al concepto de temporalidad. El tiempo, nos dice la autora, ubica una situación en relación con el momento del habla, y el aspecto se define como la manera de presentar la constitución temporal interna de una situación, esto 
es, el aspecto es entendido como la perspectiva temporal que selecciona el hablante para representar un evento. El aspecto se asocia a la gramática y al léxico. En el primer caso, se señala por la morfología verbal y es la perspectiva temporal que elige el hablante parareferirse a una situación. Indica el grado de focalización, ya sea en el de una situación en su totalidad, o en la inclusión, tanto del punto de vista inicial como el punto de vista final dela acción.

Siguiendo a ciertos autores como Vendler y Andersen, entre otros, presenta una clasificación de los verbos desde sus características semánticas en: estativos, actividades, verbos de consecución y cumplimientos, así como las distinciones semánticas inherentes en la clasificación de los verbos.

Propone, a partir de las hipótesis del aspecto y del discurso, una agenda para próximos estudios en donde se aborde la interacción entre estas dos hipótesisa partir del contexto de la narración y la expresión del tiempo y aspecto. Además, señala la necesidad de elaborar propuestas didácticas que ayuden a los alumnos a adquirir las categorías de tiempo y aspecto en lengua extranjera, con diferentes tipos de ejercicios, en cuyo diseño se consideren las diferentes etapas necesarias para la adquisición.

La aplicación de las hipótesis del aspecto y de la hipótesis del discurso, es la que presenta Beatriz Granda en el artículo "Adquisición de tiempo y aspecto en textos narrativos en español como segunda lengua".

Con relación a la hipótesis del aspecto y sigiendo a Anderson, la autora plantea que los aprendices usan, primero, la marca perfectiva con cumplimientosy realizaciones y se extienden gradualmente hacia actividades y estados. Que en lenguas con marca imperfectiva, el pasado imperfectivo aparece después que el pasado perfectivo y se restringe primero a estados y actividades, y después a realizaciones y cumplimientos. Finalmente, que la adquisición de la marca progresiva, inicialmente, se restringe a actividades y luego se extiende a realizaciones y cumplimientos.

Anota, también, quesi bien la expresión de la temporalidad es esencial a todas las lenguas, éstas difieren en lo referente a las nociones y relaciones temporales que expresan, y a la manera en que éstas son expresadas: ya sea a través del orden de palabras, recursos morfosintácticos o léxicos o a la combinación de éstos.

Cabe señalar que las definiciones que da de los verbos con relación a los tipos: estados, actividades, realizaciones y cumplimientos, complementa perfectamente a los que ya nos había presentado la Dra. Marilyn Buck.

Para realizar su investigación, la Mtra. Granda emplea la narración, la cual presenta como un modo discursivo básico de las lenguas naturales; así, desde el punto de vista lingüístico, señala, siguiendo a Dahl, que el texto narrativo es aquel en el que el hablante relata una serie de hechos reales o ficticios en el orden que tienen lugar.

Como resul tado de la investigación realizada a un grupo de 15 estudiantes de español como lengua extranjera y con 10 nativohablantes, Ia Mtra. Granda Ilega a las siguientes conclusiones: que el discurso narrativo de alumnos anglo- 
hablantes de español como $L 2$, de un nivel intermedio-avanzado de un curso formal delengua, presenta un estado del interlenguaje en el queel pretérito aparece como una forma adquirida para expresar la función del primer plano de la narración y su uso es similar al de los hablantes nativos del español.

Los resultados de esta investigación, según la autora, confirman al gunas de las hipótesis que emergen de otras investigaciones sobre la adquisición de las formas del pretérito en español como L2 y dejan abiertas nuevas hipótesis, que pueden explicar los usos particulares del discurso narrativo producido por alumnos de español cuya lengua materna es el inglés.

Lamprini Kolioussi, en su artículo "Forma y función en la adquisición de los casos gramatical es del griego" analiza la relación entreforma y función en la adquisición de los casos gramaticales del griego moderno por alumnos hispanohablantes de la UNAM. Para realizar su investigación, elige la teoría de casos, propuesta por Chomsky y sus discípulos, porque esta teoría proponeque la distribución de las frases nominales, en todas las lenguas, se puede explicar mediante la marcación del caso. En algunas lenguas, la marcación es visibley explícita, mientras queen otras no lo es.

En español, la marcación de los casos en la oración está sujeta al mismo principio que regula la aparición de las frases nominal es en las lenguas que tienen caso morfológico, pero se expresa estableciendo sus propias características y marcas de caso, como lo es, por ejemplo, la preposición de en el caso genitivo, que actúa como una categoría vacía de contenido semántico, que actúa, es decir, como una mera marca casual. Por lo tanto, en el griego moderno, los casos se manifiestan mediante las desinencias y la marcación por medio del artículo, mientras queen español no existen flexiones $y$, bajo ciertas condiciones, la marcación se registra con las preposiciones.

Los resultados de la investigación realizada por la Mtra. Koliussi a 42 alumnos degriego en el CELE y en la Facultad de Estudios Acatlán, indica que un gran porcentaje de los alumnos fallaron en la marcación morfológica del caso en dos pruebas de producción escrita, lo cual le permitió comprobar: que la hipótesis planteada era válida; que en el análisis de los datos del estudio hay evidencia de que los aprendices hispanoablantes del griego moderno interpretan en mayor porcentaje la función correcta de los casos y se manifiestan con menos competencia para codificar la formalingüística correcta en la producción escrita.

Concluye la autora proponiendo, a partir de los resultados de su investigación, una propuesta didáctica que permitaal alumno hacer conexiones correctasy estables entre la forma gramatical y su función sintáctica, que conlleve, por ende, a la enseñanza explícita de la gramática, exponiendo a los estudiantes a un inputadecuado, detal modo que sele facilite y se vuelva más obvia la conexión entre la forma y el significado de los casos.

Patricia Canovas en el artículo “El aprendizaje de vocabulario en el salón de clases: las estrategias de los estudiantes" presenta los resultados de una investigación realizada a estudiantes de 
inglés, aquí en el CELE, de nivel intermedio. El propósito fundamental de la investigación es conocer quétipo de estrategias de aprendizaje emplean los alumnos para aprender vocabulario, desde la perspectiva de dos áreas de investigación: La adquisición de lenguas y la investigación en el salón de clases.

A partir de la idea de que para adquirir vocabulario se requiere que intervengan múltiples factores, tales como la repetición, la exposición repetida de palabras, su generación en oraciones, las estrategias mnemotécnicas, la inferencia acertada y la asociación, llevan a la autora a focalizar su estudio en las estrategias de aprendizaje.

La autora emplea para su investigación en dos grupos de inglés, las taxonomías propuestas por Rebecca Oxford y Schmith, y llega a la conclusión que son sól o cuatro las estrategias de aprendizaje de vocabulario las que emplean los estudiantes con suma frecuencia para aprender palabras nuevas en el aula, a saber: el uso de L1, solicitar clarificación o verificación, solicitar el significado de una palabra, asociar con conocimientos previos. Otras estrategias empleadas con menor frecuencia son: la ubicación de pal abras nuevas en el contexto inmediato, razonar deductiva mente, conseguir ayuda, hacer uso de diccionarios. Asimismo, señala la urgencia de revalorar y actualizar el proceso de enseñanza-apren dizaje de vocabulario en general y el desarrollo de estrategias, en particular.

En el mismo tenor, Yadira Hernández en su artículo "Evaluación del conocimiento léxico de LE: tamaño y profundidad" presenta como las pruebas que miden el tamaño proveen resultados comparativos del panorama general del conocimiento léxico de los sujetos y que su inconveniente principal es que no informan que tan profundo es ese conocimiento y que, por lo tanto, no aportan datos sobresu proceso de adqui sición.

Para realizar esta tarea utiliza la Escala de Conocimiento de Vocabulario (ECV), diseñada por Paribakht y Wesche, que permite evaluar los nivel es de conocimiento de un corpusléxico. Este instrumento ayuda a capturar diferentes etapas en el desarrollo del conocimiento léxico a partir de los niveles iniciales. Cada etapa representa un aumento en el conocimiento de la pieza. De esta manera, la escala, según la concepción de sus autores, permite describir el conocimiento léxico tanto en número de piezas adquiridas (tamaño del lexicón), como en la cal idad del conocimiento acerca de cada pieza (profundidad).

La escala permite detectar pequeñas diferencias en el conocimiento léxico de diversas piezas, de esta manera, el aprendiz puede señalar: qué piezas le son completamente desconocidas, cuáles puede reconocer sin poder asignarle un significado, a cuáles puede intentar asignarles un significado sin estar totalmente seguro, a cuáles puede, con certeza, darles un significado o sinónimo y cuáles puede usar dentro de un contexto.

Después dela aplicación a 83 estudiantes el instrumento cuyo corpus estaba constituido por diez sustantivos, diez verbos y diez adjetivos, la autora del estudio señalaquela Escala deConocimiento de Vocabulario resultó ser un instrumento idóneo para capturar las etapas iniciales de proceso deadquisición deléxico, y no así para medir el tamaño del lexicón. 
Estas investigaciones dejan la discusión abierta sobre los temas expuestosy nos llevan a retomar las propuestas didácticas para la elaboración de nuevos materiales desde diferentes enfoques pedagógi cos que ayuden a los estudiantes a tomar conciencia de esos problemas que impiden una adecuada adquisición de la lengua extranjera meta.

Rosa Esther Delgadillo

CEPE-UNAM 